\title{
A multiscale quasicontinuum method for dissipative lattice models and discrete networks
}

\author{
L.A.A. Beex*, R.H.J. Peerlings, M.G.D. Geers \\ Department of Mechanical Engineering, Eindhoven University of Technology, \\ P.O. Box 513, 5600 MB Eindhoven, The Netherlands
}

\begin{abstract}
Lattice models and discrete networks naturally describe mechanical phenomena at the mesoscale of fibrous materials. A disadvantage of lattice models is their computational cost. The quasicontinuum (QC) method is a suitable multiscale approach that reduces the computational cost of lattice models and allows the incorporation of local lattice defects in large-scale problems. So far, all QC methods are formulated for conservative (mostly atomistic) lattice models. Lattice models of fibrous materials however, often require non-conservative interactions. In this article, a $\mathrm{QC}$ formulation is derived based on the virtual-power of a non-conservative lattice model. By using the virtual-power statement instead of force-equilibrium, errors in the governing equations of the force-based QC formulations are avoided. Nevertheless, the non-conservative interaction forces can still be directly inserted in the virtualpower QC framework. The summation rules for energy-based QC methods can still be used in the proposed framework as shown by two multiscale examples.
\end{abstract}

Key words: multiscale, quasicontinuum method, lattice model, discrete networks, virtual power, dissipation, plasticity

\section{Introduction}

Structural lattice models and discrete networks that include trusses or beams are frequently used to represent discrete microstructures of fibrous

\footnotetext{
${ }^{*}$ Corresponding author

Email: L.A.A.Beex@gmail.com
}

Preprint submitted to Journal of the Mechanics and Physics of Solids November 21, 2013 
materials (Bronkhorst, 2003; Clyne et al., 2005; Zohdi and Powell, 2006; Stylianopoulos and Barocas, 2007; Ridruejo et al., 2010; Silberstein et al., 2012; Beex et al., 2013; Wilbrink et al., 2013). Likewise, for investigating the mechanical responses of other materials, e.g. concrete and polymers, lattice models and discrete networks are often applied (Lilliu and Van Mier, 2003; Cusatis et al., 2003; Ostoja-Starzewski and Wang, 2006; Rinaldi et al., 2008; Kim and Buttlar, 2009; Zhao, 2012). The advantage of discrete models is that they naturally incorporate discrete phenomena occurring in meso- and microstructures of many materials. Individual microscale events such as fiber failure and bond failure, precursors for macroscale failure, can be readily incorporated in lattice models, whereas these they are not easily included in continuum models. Even the incorporation of global mechanisms such as large rotations may lead to relatively complex continuum models and finite element implementations (Peng and Cao, 2005; Ten Thije et al., 2007), whereas they are naturally captured by truss networks.

A clear disadvantage of lattice models is the required computational effort for large-scale physically relevant models. This large computational cost essentially results from the construction of the lattice model at the meso- or microscale. Consequently, if lattice models are being used for macroscopic computations, a large number of lattice points is involved. First, this leads to a large number of degrees of freedom (DOFs) making the system of equations computationally expensive to solve. Secondly, the construction of the system of equations is computationally expensive because all lattice points must be visited for this (Beex et al., 2011).

A suitable approach to reduce the computational cost of structural lattice models is the quasicontinuum (QC) method. The QC method was originally developed for the reduction of atomistic lattice models by Tadmor et al. (1996a) and has been widely used to investigate phenomena such as intergranular fracture (Miller et al., 1998) and nanoindentation (Tadmor et al., 1996b; Knap and Ortiz, 2001; Kulkarni et al., 2008). In a previous study (Beex et al., 2011) the applicability of the QC method has been demonstrated for structural lattice models of fibrous materials that employ elastic trusses. An overview of several QC frameworks is given by Miller and Tadmor (2002).

The benefit of the QC method is its intrinsic multiscale character, allowing the accurate incorporation of local lattice defects in large-scale problems. This is not trivially possible with multiscale methods that are based on computational homogenization (Stylianopoulos and Barocas, 2007). Moreover, the QC method entirely relies on the microstructural lattice topology, unlike 
approaches as those of Xiao and Belytschko (2004) and Fish et al. (2007), that need a continuum description in addition to the discrete model. This is an advantage, since continuum descriptions for fibrous materials tend to be complex to formulate (Peng and Cao, 2005). Another advantage is that a number of QC frameworks (Knap and Ortiz, 2001; Eidel and Stukowski, 2009; Beex et al., 2011, 2012a) do not require a handshaking region or coupling procedure between fully resolved domains (in which the exact lattice model is recovered) and coarse domains (in which an approximation is made). Several other multiscale approaches require such a coupling procedure (Shenoy et al., 1999; Xiao and Belytschko, 2004; Fish et al., 2007; Ha-Minh et al., 2011).

The QC method introduces two remedies to reduce the computational cost of lattice models. First, interpolation is applied to the displacements of the lattice points to reduce the number of DOFs and thus reducing the size of the governing set of equations. In coarse regions, the interpolation triangles are large so that many lattice points are interpolated. In regions of interest, e.g. around lattice defects, the exact lattice model is captured by refining the interpolation such that every lattice point corresponds to a point of the interpolation triangulation (so-called representative point or reppoint).

Secondly, so-called summation rules are used in the QC method to ensure that only a small, selective number of lattice points (so-called sampling points) needs to be visited to construct the governing equations, instead of all lattice points. To obtain an accurate solution, all lattice points in the fully resolved regions are sampling points, while in the coarse regions only a small number of points is used (see for instance the studies of Zhang and Gunzburger (2010); Beex et al. (2011) and Beex et al. (2012a)).

Most QC formulations are based on minimizing the total potential energy of the interpolated system (Tadmor et al., 1996a; Miller et al., 1998; Shenoy et al., 1999; Eidel and Stukowski, 2009). Also the QC formulation of Beex et al. (2011), developed for structural lattice models with elastic interactions only, uses this ansatz. Depending on the application, such an elastic description is adequate (Delincé and Delannay, 2004; Sharma and Sutcliffe, 2004; Gonella and Ruzzene, 2008; Hatami-Marbini and Picu, 2009; Zeman et al., 2011). For many applications however, more advanced descriptions of lattice interactions are required that include dissipation in the lattice interactions by e.g. using plasticity (Kato et al., 1997; Desphande et al., 2001; Arnoux et al., 2002; Bronkhorst, 2003; Chen and Baker, 2003; Mohr, 2005; Doyoyo and $\mathrm{Hu}, 2006$; Silberstein et al., 2012; Beex et al., 2013; Wilbrink et al., 2013) or damage (Cusatis et al., 2003). In these cases, a straightforward minimiza- 
tion of the potential energy can no longer be employed since the dissipation leads to non-conservative interactions. Energy-based QC formulations are thus inadequate for structural lattice models with dissipative interactions.

Existing force-based QC formulations may be appropriate alternatives. The QC formulations that depart from a force-equilibrium however (Knap and Ortiz, 2001; Kulkarni et al., 2008), also appear to be energetically inconsistent for conservative systems. This has been shown by Eidel and Stukowski (2009) for the widely used cluster QC approach of Knap and Ortiz (2001).

The aim of this study is therefore to develop an energetically consistent QC formulation for non-conservative lattice models. Our point of departure for this forms a virtual-power statement (Germain, 1973) in which the nonconservative forces can be directly inserted. As a result, the framework is equivalent to energy minimization for conservative lattices, but its applicability is broader.

Another advantage of using the virtual-power statement is that summation rules proposed in energy-based QC formulations can directly be used in the proposed QC methodology. In this study, the summation rule for atomistic lattice models as presented by Beex et al. (2012a) is used to reduce the computational efforts of the considered lattice model.

The outline of this paper is as follows. In section 2 the thermodynamics of structural lattice models with dissipative interaction forces are formulated. The lattice model of interest is discussed here as well. In section 3, the virtual-power-based QC formulation is introduced and applied to an elastoplastic lattice model consisting of an equidistant X-braced truss network with elastoplastic interactions. This fairly simple lattice model is chosen in order to show the possibilities of the framework. In section 4 two multiscale numerical examples are simulated to evaluate the accuracy and efficiency of the virtual-power-based QC method and to illustrate some of key features for fibrous materials.

\section{Structural lattice models with non-conservative interactions}

It is useful to formulate structural lattice models, as adopted in the QC method, on a thermodynamic basis. The reason is that most QC methods are based on energy-minimization and that thermodynamical inconsistencies in sampling points are carried over to their corresponding lattice points. These inconsistenties can significantly contribute to the inaccuracy of the QC method (Eidel and Stukowski, 2009). The thermodynamics of dissipative 
lattice models are therefore first considered. We subsequently particularize the framework to the case of a lattice of elastoplastic trusses and briefly discuss solution methods for it.

The lattice model studied in this paper is a two-dimensional equidistant X-braced network of elastoplastic trusses (see Fig. 1). It can be considered as a discrete representation of the microstructure of a fictitious fibrous material. A unit cell of the truss network contains four truss nodes positioned at the locations where the horizontal, vertical and diagonal trusses meet (see Fig. 1). At the center of a unit cell, where the diagonals cross each other, no truss node is present. This means that every truss node in the network is connected to eight other nodes. The considered simple lattice is esentially used to show the possibilities of the virtual-power-based QC method for structural lattice models including dissipative mechanisms.

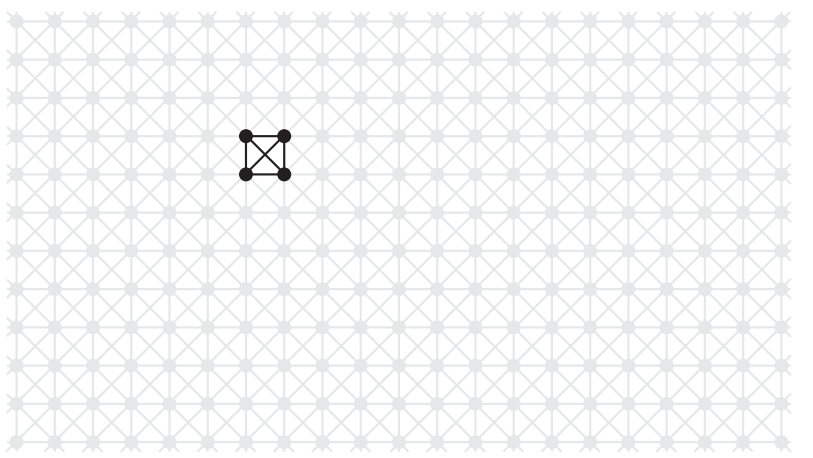

Figure 1: The equidistant $\mathrm{X}$-braced lattice model with elastoplastic trusses as considered in this paper. A unit cell of the truss network is marked in black.

\subsection{Thermodynamics of non-conservative lattice models}

The lattice model considered here merely contains trusses. The DOFs of the system are thus only the displacement components of the lattice nodes. For a solution of the lattice model, the internal power of the lattice equals the external power:

$$
\dot{\mathbf{u}}^{T i n t} \mathbf{F}=\dot{\mathbf{u}}^{T \text { ext }} \mathbf{F} \quad \forall \dot{\mathbf{u}}
$$

where $\mathbf{u}$ refers to the column matrix containing the components of the displacement vectors of all $n$ lattice points. The column matrices ${ }^{i n t} \mathbf{F}$ and ${ }^{e x t} \mathbf{F}$ 
contain the components of the resulting internal forces and externally applied forces. In Eq. (1) $\dot{\mathbf{u}}^{T}{ }^{\text {int }} \mathbf{F}$ can be identified as the internal power, ${ }^{\text {int }} P$, and $\dot{\mathbf{u}}^{T}{ }^{e x t} \mathbf{F}$ as the external power, ${ }^{e x t} P$.

A dissipative lattice model depends not only on a set of the (controllable) kinematic variables, $\mathbf{u}$, but also on a set of (not directly controllable) internal variables, stored in column matrix $\mathbf{z}$. The size of this column matrix is $Z \times 1$ and is independent of the number of kinematic variables, $2 n$, since it depends on the number of dissipation mechanisms in the lattice. The energy stored in the lattice, $E$, thus depends on the kinematic and internal variables, i.e. $E=E(\mathbf{u}, \mathbf{z})$. For a lattice model, the total energy stored in the lattice, $E$, can be written in terms of the stored energy per lattice point $i, E^{i}$, which can be written in terms of the energy stored per interaction (truss) between a lattice point, $i$, and one of its neighbors, $j, E^{i j}$ :

$$
E(\mathbf{u}, \mathbf{z})=\sum_{i=1}^{n} E^{i}(\mathbf{u}, \mathbf{z})=\sum_{i=1}^{n} \sum_{j \in B^{i}} \frac{1}{2} E^{i j}(\mathbf{u}, \mathbf{z})
$$

where $B^{i}$ contains the neighboring points of lattice point $i$, which is a subset of the index set $N=\{1, \ldots, n\}$ containing all $n$ lattice points $\left(B^{i} \subseteq N\right)$. The factor $1 / 2$ arises because half of the energy $E^{i j}$ associated with the interaction between $i$ and $j$ is attributed to node $i$ and the other half to $j$. Using the chain rule, the rate of the stored energy reads:

$$
\dot{E}=\dot{\mathbf{u}}^{T} \frac{\partial E}{\partial \mathbf{u}}+\dot{\mathbf{z}}^{T} \frac{\partial E}{\partial \mathbf{z}}
$$

Now the internal power and the rate of the stored energy have been defined, the first law of thermodynamics can be used to relate them and determine the dissipation. The first law states that the power performed by a system is the sum of the rate of the stored energy and the rate of dissipation of the system:

$$
{ }^{\text {int }} P=\dot{E}+\dot{D}
$$

where $\dot{D}$ is the rate of dissipation. Substituting Eq. (1 \& 3) in Eq. (4) yields $\dot{D}$ as: 


$$
\dot{D}=\dot{\mathbf{u}}^{T}\left({ }^{i n t} \mathbf{F}-\frac{\partial E}{\partial \mathbf{u}}\right)-\dot{\mathbf{z}}^{T} \frac{\partial E}{\partial \mathbf{z}} .
$$

Similar to the work of (Germain, 1973), it is now assumed that no dissipation takes place if the internal history variables, $\mathbf{z}$, which are related to the dissipation mechanisms, remain constant $(\dot{D}=0$ if $\dot{\mathbf{z}}=\mathbf{0})$. To ensure that this requirement is valid for all (controllable) $\dot{\mathbf{u}}$, the term between brackets in Eq. (5) should vanish. The standard expression for the internal forces results:

$$
{ }^{i n t} \mathbf{F}=\frac{\partial E}{\partial \mathbf{u}}
$$

and the rate of dissipation is reduced to:

$$
\dot{D}=-\dot{\mathbf{z}}^{T} \frac{\partial E}{\partial \mathbf{z}}
$$

Furthermore, the second law of thermodynamics states that the entropy of a system must remain constant or increase, i.e. $\dot{D} \geq 0$. If we now introduce a column matrix with dissipative forces ${ }^{z} \mathbf{F}=-\frac{\partial E}{\partial \mathbf{z}}$, the following expression is obtained for the dissipation rate:

$$
\dot{D}=\dot{\mathbf{z}}^{T z} \mathbf{F} \geq 0
$$

A dissipation potential, $\Phi$, needs to be formulated as functions of the kinematic and internal variables ( $\mathbf{u}$ and $\mathbf{z}$ respectively). The dissipation potential can be summed from the dissipation potential of each lattice point, $\Phi^{i}$, (if dissipation takes place in the lattice points) or summed from the dissipation potential of each lattice interaction, $\Phi^{i j}$, (if dissipation takes place in the interactions):

$$
\Phi(\mathbf{u}, \mathbf{z})=\sum_{i=1}^{n} \Phi^{i}(\mathbf{u}, \mathbf{z})=\sum_{i=1}^{n} \sum_{j \in B^{i}} \frac{1}{2} \Phi^{i j}(\mathbf{u}, \mathbf{z})
$$


Note that this expression entails that each interaction is visited twice (one node on each side), as is performed for the stored energy in Eq. (2).

At this point, the thermodynamical setting for a dissipative lattice model with trusses is complete, except for the formulation of the stored energy per truss and the formulation of the dissipation potential per point or interaction as functions of the kinematic, $\mathbf{u}$, and internal variables, $\mathbf{z}$. Both formulations depend on the specific lattice model of interest. For a lattice with elastoplastic trusses (see Fig. 1), the expressions of the energy stored per truss, $E^{i j}$ in Eq. (2), and the dissipation potential for a truss, $\Phi^{i j}$ in Eq. (9), (since dissipation takes place in the trusses for a network with elastoplastic trusses) are established below.

\subsection{Elastoplastic interactions}

The formulations of the stored energy per truss and the dissipation potential per truss are derived here for a truss that is connected to lattice point $i$ and neighboring lattice point $j$. However, the superscripts $i j$ are dropped below for ease of notation.

The elastoplastic trusses in the considered lattice are all of the same material with the same material description. The elastic part of the material description is linear, whereas the hardening behavior is yet to be defined

(in the dissipation potential, $\Phi)$. First the stored energy per truss $\left(E^{i j}\right.$ in Eq. (2)) is defined in terms of $\mathbf{u}$ and $\mathbf{z}$.

The total axial strain of one elastoplastic truss, $\epsilon$, is split in an elastic part, ${ }^{e} \epsilon$, and a plastic part, ${ }^{p} \epsilon$, as follows:

$$
{ }^{e} \epsilon={ }^{e} \epsilon+{ }^{p} \epsilon \text {. }
$$

The total strain is taken linear in terms of the elongation, $\lambda$ :

$$
\epsilon=\lambda-1
$$

The elongation depends on the kinematic variables, $\mathbf{u}$, according to:

$$
\lambda=\frac{\left|\vec{x}^{j}+\vec{u}^{j}-\vec{x}^{i}-\vec{u}^{i}\right|}{\left|\vec{x}^{j}-\vec{x}^{i}\right|}
$$


where $\vec{u}^{i}$ and $\vec{u}^{j}$ are the displacement vectors of nodes $i$ and $j$ respectively. The original length of the interaction is represented by $\left|\vec{x}^{j}-\vec{x}^{i}\right|$ where $\vec{x}^{i}$ is the original position vector of node $i$. The internal variables, $\mathbf{z}$, are taken as the plastic strains of all trusses, i.e. ${ }^{p} \epsilon=z$. By inserting ${ }^{p} \epsilon=z$ and Eq. (11 \& 12) in Eq. (10), the following expression is obtained for the elastic strain:

$$
{ }^{e} \epsilon=\frac{\left|\vec{x}^{j}+\vec{u}^{j}-\vec{x}^{i}-\vec{u}^{i}\right|}{\left|\vec{x}^{j}-\vec{x}^{i}\right|}-1-z .
$$

This relation relates the elastic strain of a truss, ${ }^{e} \epsilon$, to $\mathbf{u}$ and $\mathbf{z}$.

The elastic part of the material description of all trusses is linear. The stored energy of a truss can then be expressed in terms of ${ }^{e} \epsilon$, according to:

$$
E=\frac{1}{2} Y A\left|\vec{x}^{j}-\vec{x}^{i}\right|{ }^{e} \epsilon^{2}
$$

where $Y$ represents the Young's modulus and $A$ the original cross-sectional area, which is remains constant for all elongation. Insertion of Eq. (13) in Eq. (14) leads to the following expression:

$$
E=\frac{1}{2} Y A\left|\vec{x}^{j}-\vec{x}^{i}\right|\left(\frac{\left|\vec{x}^{j}+\vec{u}^{j}-\vec{x}^{i}-\vec{u}^{i}\right|}{\left|\vec{x}^{j}-\vec{x}^{i}\right|}-1-z\right)^{2} .
$$

Now we can define the expression for the local potential dissipation for each truss ( $\Phi^{i j}$ in Eq. (9), although below we drop superscript $i j$ again). The following formulation is regularly used to describe the onset of yielding in a $1 \mathrm{D}$ system (the axial direction of a truss):

$$
\Phi=\left|{ }^{z} F\right|-{ }^{y} F(\xi) \leq 0
$$

with

$$
{ }^{z} F=-\frac{\partial E}{\partial z}
$$


where ${ }^{y} F$ is the yield function. This expression entails that no dissipation (plastic deformation) occurs if the force ${ }^{z} F$ remains below the corresponding yield force, ${ }^{y} F$. The full dissipation potential in Eq. (9) depends on the column matrix ${ }^{z} \mathbf{F}$, but each local term of the dissipation potential only depends on one component of ${ }^{z} \mathbf{F}$, namely ${ }^{z} F$. Therefore each local term can be locally determined, as commonly done in elastoplastic descriptions. The internal history variable $\xi$, which for all trusses are stored in column matrix $\boldsymbol{\xi}$, is introduced for one truss by:

$$
\dot{z}=\dot{\xi} \frac{\partial \Phi}{\partial^{z} F}=\dot{\xi} \operatorname{sign}\left({ }^{z} F\right)
$$

To ensure a non-negative rate of dissipation, the following Kuhn-Tucker equations are imposed for each truss:

$$
\dot{\xi} \geq 0 \quad \Phi \leq 0 \quad \dot{\xi} \Phi=0 \text {. }
$$

The internal history variable $\xi$ is thereby the effective plastic strain of a truss. Note that $\operatorname{since} \operatorname{sign}(z)=\operatorname{sign}\left({ }^{z} F\right)$, Eq. (8) is satisfied for each individual truss (i.e. each component of $\dot{\mathbf{z}}$ and ${ }^{z} \mathbf{F}$ ) and thus for the entire lattice.

For the hardening behavior of a truss in the lattice the following formulation is used:

$$
{ }^{y} F=A^{o} \sigma\left|\vec{x}^{j}-\vec{x}^{i}\right|\left(1+H \xi^{\alpha}\right),
$$

where $H$ and $\alpha$ are hardening parameters and ${ }^{\circ} \sigma$ is the initial yield stress. The same values are used for all trusses.

\subsection{Solution procedure}

The solution of the lattice can be found by solving Eq. (1) while taking into account the inequality constraints in Eq. (19) for all trusses. This system of equations is non-linear due to the non-linear material description of the trusses (see Eq. (20)) and the fact that we allow for large rotations (see Eq. (15)). A Newton-Raphson procedure can be used to solve Eq. (1), for which a first-order Taylor expansion is required: 


$$
\dot{\mathbf{u}}^{T}\left({ }^{i n t} \mathbf{F}\left({ }^{*} \mathbf{u},{ }^{*} \mathbf{z}\right)+\mathbf{K}\left({ }^{*} \mathbf{u},{ }^{*} \mathbf{z}\right) d \mathbf{u}\right)=\dot{\mathbf{u}}^{T}{ }^{e x t} \mathbf{F} \quad \forall \dot{\mathbf{u}},
$$

where ${ }^{*} \mathbf{u}$ and ${ }^{*} \mathbf{z}$ are the displacement components and the plastic strains of the previous iteration respectively. The correction to the displacement components computed in the present iteration is represented by $d \mathbf{u}$. The overall stiffness matrix $\mathbf{K}$ is defined here as $\frac{\partial^{i n t} \mathbf{F}}{\partial \mathbf{u}}$ and is thus a second order derivative of the stored energy (since ${ }^{i n t} \mathbf{F}$ is the first order derivative of the stored energy, see Eq. (6)). Note that the inequality constraints (see Eq. (19) for the constraints acting in each truss) must be met as well, which can be checked locally for each truss of the network.

In the Newton-Raphson scheme, the first expression in Eq. (21) is used to obtain a new estimate of the displacement components, whereas the new plastic strains are determined from these newly obtained displacements by the use of the inequality constraints in the second expression in Eq. (21). The latter are numerically dealt with by a standard return mapping procedure (Wilkins, 1963; Simo and Taylor, 1986). Since each inequality constraint is related to one local dissipation mechanism (in each truss), each inequality constraint is treated individually in the solution procedure.

Although not incorporated in Eq. (21), the boundary conditions of the system must be taken into account. Neumann boundary conditions are present in the external force column, ${ }^{e x t} \mathbf{F}$, and the Dirichlet boundary conditions are incorporated in the displacement column, u. After the system is partitioned in a standard manner, the incremental solution results for the standard Newton-Raphson procedure.

The system of equations is computationally expensive to solve for largescale lattice models (i.e. $n$ is large). A large number of $2 n$ DOFs (displacement components) is involved, making the first expression in Eq. (21) computationally expensive to solve. Moreover, all $n$ lattice points need to be visited to construct the system of equations in Eq. (21), according to Eq. (2, $6 \& 9)$.

\section{Virtual-power-based quasicontinuum method}

The QC method, developed for (conservative) atomistic lattice models (Tadmor et al., 1996a), introduces two remedies to improve the efficiency of direct lattice computations. First, interpolation of the displacements of the 
lattice points ensures that the number of displacement components (DOFs) is reduced. Furthermore, summation is used in the QC method to achieve a more efficient construction of the governing equations. Instead of using all lattice points to construct the governing equations, an estimate of the governing equations is obtained by selecting only a small number of lattice points.

\subsection{Interpolation}

Interpolation is introduced in QC methods by imposing an interpolation triangulation to a lattice model. For the two-dimensional lattice model considered here, an example of a triangulation is shown in Fig. 2. Nodes of the triangles are positioned at chosen lattice points and the displacements of the remaining lattice points are interpolated between them. Linear interpolation is generally used as in this paper, except by Kwon et al. (2009). As a result of the interpolation, the displacements of the interpolated lattice points follow directly from the displacements of the lattice points at triangle nodes. The latter are therefore referred to as representative points or reppoints (or repatoms if the method is being used for atomistic lattices). The reppoints, stored in set $R$, are selected from all lattice points, i.e. $R \subseteq N$.

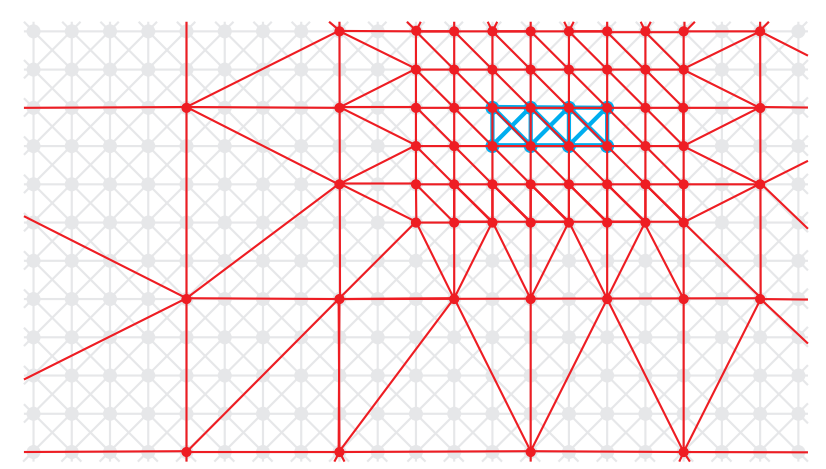

Figure 2: The X-braced truss network (grey) with an imposed triangulation (red). The blue bold trusses in the center of the fully resolved region are substantially stiffer than the remaining grey trusses.

The use of interpolation of the lattice model ensures that the QC method is a true multiscale method. Consider for instance Fig. 2 in which a number of trusses is significantly stiffer than the regular trusses. In the region surrounding the stiff trusses significant displacement fluctuations are expected. 
Therefore, a fully resolved interpolation grid is used there to capture these fluctuating displacements, i.e. every lattice point is a reppoint. The exact lattice model is recovered here, while in the remaining regions the lattice model is interpolated and many DOFs are eliminated. As a consequence, the lattice points displace in an affine manner in these coarse domains. Coarse domains (large triangles) are therefore only allowed to be used in regions with smooth displacement fluctuations to ensure sufficient accuracy. The fact that in coarse domains the displacements are coarse-grained and in the fully resolved domains the displacements of the individual lattice points are incorporated, gives the QC method its multiscale character.

The displacement components of all lattice points, $\mathbf{u}$, are affected by interpolation, resulting in condensed displacement column $\tilde{\mathbf{u}}$, which can be expressed as a function of the displacement components of the reppoints by the following expression:

$$
\mathbf{u} \approx \tilde{\mathbf{u}}=\Psi^{r} \mathbf{u}
$$

where ${ }^{r} \mathbf{u}$ is the column matrix with the displacement components of the reppoints of size $2 r \times 1$ for the two-dimensional truss network of interest. Here, $r$ refers to the number of reppoints. The condensation matrix is denoted by $\Psi$ and its size is $2 n \times 2 r$. It contains the values of the interpolation functions, evaluated at the positions of all lattice points.

Interpolation directly affects the displacement components, $\mathbf{u}$, of the lattice according to Eq. (22). The interpolated displacements are $C_{0}$-continuous as a result of the linear interpolation. According to the inequality constraints in Eq. (21), the internal variables follow from the displacements. Consequently, they are only indirectly influenced by the interpolation of $\mathbf{u}$. The internal variables are (approximately) constant within an interpolation triangle as a consequence of the linear interpolation of the displacements, since they are defined as (plastic) strains, of the order of the gradients of the displacements (kinematic variables).

By substituting Eq. (22) in Eq. (21) the formulation of the virtual-power statement reads:

$$
{ }^{r} \dot{\mathbf{u}}^{T}\left(\boldsymbol{\Psi}^{T}{ }^{i n t} \mathbf{F}\left({ }^{*} \tilde{\mathbf{u}},{ }^{*} \mathbf{z}\right)+\Psi^{T} \mathbf{K}\left({ }^{*} \tilde{\mathbf{u}},{ }^{*} \mathbf{z}\right) \Psi d^{r} \mathbf{u}\right)={ }^{r} \dot{\mathbf{u}}^{T} \boldsymbol{\Psi}^{T}{ }^{e x t} \mathbf{F} \quad \forall^{r} \dot{\mathbf{u}},(23)
$$


where $\Psi^{T \text { int }} \mathbf{F}$ and $\Psi^{T} \mathbf{K} \boldsymbol{\Psi}$ can be identified as the condensed internal force column, ${ }^{i n t} \tilde{\mathbf{F}}$ (of size $2 r \times 1$ ), and the condensed stiffness matrix, $\tilde{\mathbf{K}}$ (of size $2 r \times 2 r)$, respectively. For the sake of clarity the condensed external force column is not considered in more detail here and below. The condensed counterparts of the internal force column and stiffness matrix are formulated as follows:

$$
\begin{gathered}
{ }^{i n t} \tilde{\mathbf{F}}=\Psi^{T} \sum_{i=1}^{n} \sum_{j \in B^{i}} \frac{1}{2} \frac{\partial E^{i j}}{\partial \mathbf{u}} \\
\tilde{\mathbf{K}}=\Psi^{T} \sum_{i=1}^{n} \sum_{j \in B^{i}} \frac{1}{2} \frac{\partial^{2} E^{i j}}{\partial \mathbf{u}^{2}} \Psi .
\end{gathered}
$$

Note that the constraints in all trusses (see Eq. (19) for the constraints acting in one truss) must still be met in in the condensed system.

To ensure that the condensed governing equations in Eq. (23) adequately approach the original governing equations in Eq. (21), coarse domains (i.e. large triangles) may only exist in regions with small displacement fluctuations. In these regions, the displacements are linearly interpolated resulting in an equal virtual power of two neighboring lattice points in one triangle $\left({ }^{r} \dot{\mathbf{u}}^{T} \boldsymbol{\Psi}^{T} \frac{\partial E^{i}}{\partial \mathbf{u}}={ }^{r} \dot{\mathbf{u}}^{T} \boldsymbol{\Psi}^{T} \frac{\partial E^{i+1}}{\partial \mathbf{u}}\right)$. This is only valid if the virtual powers of these two lattice points in the uncondensed system are practically identical $\left(\dot{\mathbf{u}}^{T} \frac{\partial E^{i}}{\partial \mathbf{u}} \approx \dot{\mathbf{u}}^{T} \frac{\partial E^{i+1}}{\partial \mathbf{u}}\right)$.

As a result of Eq. (23) and because $r \ll n$, the (condensed) system in Eq. (23) is substantially more efficient to solve than the (uncondensed) system in Eq. (21). This is in correspondence with other QC methods based on conservative lattices. The inequality constraints for the entire network, which originate from the considered elastoplastic (non-conservative) lattice, remain unaffected by interpolation. Through interpolation, a new estimate of the displacements is computationally more efficiently obtained. Yet, all constraints need to be satisfied individually and still all $n$ lattice points need to be visited for the construction of the governing equations in Eq. (23).

\subsection{Summation}

Now the solution space of the system is reduced by means of interpolation, the first cause of the large computational effort has been addressed. However, 
still all $n$ lattice points must be visited to construct the condensed internal force column, ${ }^{i n t} \tilde{\mathbf{F}}$, and the condensed stiffness matrix, $\tilde{\mathbf{K}}$.

The remedy adopted in the QC method to avoid accessing all $n$ lattice points is to access only a small number of $s$ lattice points in order to obtain an estimate of the condensed virtual power (i.e. the condensed governing equations). This procedure is called summation in QC methodologies. The lattice points that are used for this are referred to as sampling points since they sample the virtual power of the lattice points in its vicinity. They are stored in set $S$ and are selected from all lattice points $(S \subseteq N)$.

The key principle of summation is that the virtual power of a sampling point $i$ can be used to represent the virtual power of $w^{i}$ lattice points in the vicinity of the sampling point (including sampling point $i$ itself). This entails for the governing equations that:

$$
{ }^{r} \dot{\mathbf{u}}^{T}\left({ }^{i n t} \tilde{\overline{\mathbf{F}}}\left({ }^{*} \tilde{\mathbf{u}},{ }^{*} \mathbf{z}\right)+\tilde{\overline{\mathbf{K}}}\left({ }^{*} \tilde{\mathbf{u}},{ }^{*} \mathbf{z}\right) d^{r} \mathbf{u}\right)={ }^{r} \dot{\mathbf{u}}^{T} \boldsymbol{\Psi}^{T}{ }^{e x t} \mathbf{F} \quad \forall^{r} \dot{\mathbf{u}} .
$$

with

$$
\begin{aligned}
& { }^{i n t} \tilde{\overline{\mathbf{F}}}=\boldsymbol{\Psi}^{T} \sum_{i \in S} w^{i} \sum_{j \in B^{i}} \frac{1}{2} \frac{\partial E^{i j}}{\partial \mathbf{u}}, \\
& \tilde{\overline{\mathbf{K}}}=\Psi^{T} \sum_{i \in S} w^{i} \sum_{j \in B^{i}} \frac{1}{2} \frac{\partial^{2} E^{i j}}{\partial \mathbf{u}^{2}} \boldsymbol{\Psi},
\end{aligned}
$$

in which ${ }^{i n t} \tilde{\overline{\mathbf{F}}}$ and $\tilde{\overline{\mathbf{K}}}$ are the summed condensed internal force column and summed condensed stiffness matrix, respectively.

As a result of the presence of the weight factor, $w^{i}$, the dissipation potential will not show the same elastoplastic behavior for a truss connected to a sampling point. To ensure that the elastoplastic behavior of a truss recovers its response in the direct lattice model (i.e. to ensure equal dissipation), the dissipation potential for the truss between sampling point $i$ and neighbor $j$ in Eq. (16), $\Phi$, needs to be modified to (where we use the superscript $i j$ again for clarity): 


$$
\tilde{\tilde{\Phi}}^{i j}=\left|z^{i j} \tilde{\bar{F}}\right|-w^{i y} F\left(\xi^{i j}\right) \leq 0,
$$

since ${ }^{i j} \tilde{\bar{F}}=w^{i} \frac{\partial E^{i j}}{\partial z^{i j}}$, where $z^{i j}$ refers to the component of $\mathbf{z}$ that is associated with the truss between points $i$ and $j$. Eq. (29) implies that the yield function ${ }^{y} F$ is to be weighted equally with the same weight factor $w^{i}$.

The advantage of using summation is that only a small number of $s$ sampling points (assuming that $s \ll n$ ) need to be visited to obtain an approximation of the condensed governing equations, instead of all $n$ lattice points (to determine the condensed governing equations exactly). In this way, the QC method not only solves the governing equations efficiently by interpolation but also efficiently construct the governing equations. Since the inequality constraints hold for each truss connected to a sampling point, the inequality constraints apply to all trusses related to sampling points.

An important advantage of the proposed virtual-power based QC framework is that the internal forces are accurately related to all virtual displacements of influence. This automatically follows from the virtual-power based framework as was the case for the energy-based QC frameworks (Eidel and Stukowski, 2009; Beex et al., 2011) which did not take dissipation into account. The relation between the virtual displacements and the forces is for instance illustrated by the summed condensed internal force column in Eq. (27) in which $\frac{\partial}{\partial \mathbf{u}}$ ensures that stored energy of a truss, represented by $E^{i j}$, is related to the virtual displacements of the sampling point itself and also to its neighboring points.

For the summed condensed internal force column in a force-based QC framework (Knap and Ortiz, 2001) however, the following formulation is used:

$$
{ }^{i n t} \tilde{\overline{\mathbf{F}}}=\Psi^{T} \sum_{i \in S}\left(\left(w^{i} \sum_{j \in B^{i}} \frac{\partial E^{i j}}{\partial \mathbf{u}^{i}}\right)^{T} \mathbf{N}^{i}\right)^{T}
$$

where for the two-dimensional lattice considered here $\mathbf{u}^{i}$ contains the displacement components of sampling point $i\left(\mathbf{u}^{i}=\left[\begin{array}{ll}u_{2 i-1} & u_{2 i}\end{array}\right]^{T}\right)$ and $\mathbf{N}^{i}$ is of size $2 \times 2 n$. The components of $\mathbf{N}^{i}$ are given by:

$$
N_{k l}^{i}= \begin{cases}1 & \text { if }(k=1 \& l=2 i-1) \text { or }(k=2 \& l=2 i) \\ 0 & \text { otherwise. }\end{cases}
$$


Eq. (30) illustrates that only the dependence of the stored energy of a truss, $E^{i j}$, on the displacement components of the sampling points are considered in the force-based methods (indicated by $\frac{\partial}{\partial \mathbf{u}^{i}}$ ), but not its dependence on the displacement components of the neighboring points of the sampling points. As a consequence, the accuracy of force-based QC methods is compromized, whereas this is not the case for the proposed virtual-power-based QC method in which the same dependence is recovered as in energy-based QC methodologies. This is explained extensively by Eidel and Stukowski (2009) for conservative lattice models.

Now the question arises which lattice points are suitable to serve as sampling points. The answer to this question results from the requirement that the virtual power of each sampling point needs to be approximately the same as those of the lattice points represented by each sampling points. Hence, we need to identify lattice points for which ${ }^{r} \dot{\mathbf{u}}^{T} \Psi^{T} \frac{\partial E^{i}}{\partial \mathbf{u}} \approx{ }^{r} \dot{\mathbf{u}}_{r}^{T} \Psi^{T} \frac{\partial E^{i+1}}{\partial \mathbf{u}}$, so that one of them can serve as a sampling point to represent the others. The number of lattice points that each sampling point $i$ represents, the weight factor $w^{i}$, can be determined in different ways and one has to decide if the virtual power of the sampling points is established in a local or nonlocal fashion. The combination of these three issues (which sampling points to select, how to determine $w^{i}$ and which sampling points are treated locally or nonlocally) is specified in a so-called summation rule of which several have been proposed (Tadmor et al., 1996a; Knap and Ortiz, 2001; Eidel and Stukowski, 2009; Zhang and Gunzburger, 2010; Beex et al., 2011, 2012a). An overview is given by Miller and Tadmor (2002).

Although the summation rule presented by Beex et al. (2011) has been developed for structural lattice models with only nearest-neighbor interactions (as the truss network considered in this study) the modified version of this summation rule (Beex et al., 2012a), the so-called central summation rule, is used here. The reason is that less sampling points are necessary in the central summation rule which leads to an efficient QC method. A disadvantage of the central summation rule compared to the original summation rule (Beex et al., 2011) is that it only gives an estimate of virtual power. As shown by Beex et al. (2012a) and below in section 4, an improved accuracy in the fully resolved regions of interest can be obtained by increasing the size of the fully resolved regions.

In the central summation rule one internal sampling point is selected in the center of each triangle to sample all lattice points in the triangle and on top of the triangle edges (with $w^{i} \geq 1$ ) while the reppoints of the triangle are 
selected to only represent themselves (see Fig. 3). The reppoints are selected as discrete sampling points (with $w^{i}=1$ ) so that in the fully resolved regions the exact lattice model is recovered. In case all lattice points in a triangle are located at triangle edges and triangle nodes, no internal sampling node is selected and all lattice points of the corresponding triangle are selected as discrete sampling nodes to overcome metastable solutions (Beex et al., 2012a). Furthermore, the virtual powers of all sampling points are computed in a nonlocal fashion so that no internal interface occurs, as for instance in the QC methods of Tadmor et al. (1996a) and Shenoy et al. (1999) and other multiscale approaches of Xiao and Belytschko (2004) and Fish et al. (2007).

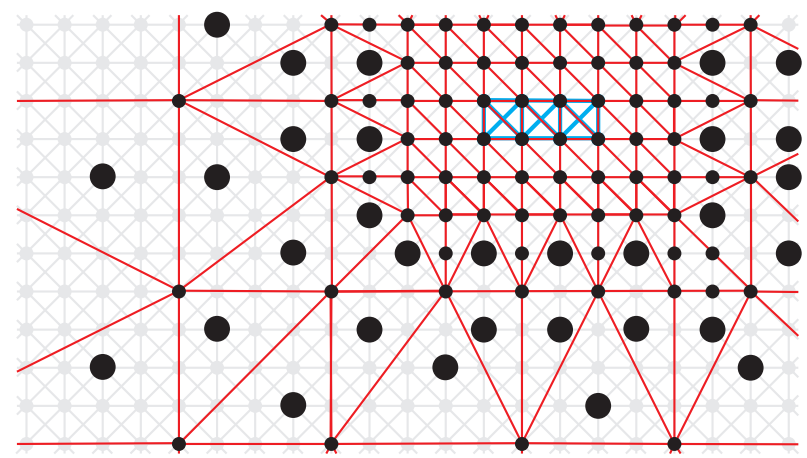

Figure 3: Schematic representation of the central summation rule applied to the X-braced truss network. The thick, blue trusses in the fully resolved region are substantially stiffer than the regular trusses. Black dots are discrete sampling points (with $w^{i}=1$ ) and large black dots are internal sampling points (with $w^{i} \leq 1$ ). The neighboring nodes of the sampling nodes that must be taken into account in the nonlocal computation are not highlighted.

\section{Numerical examples}

In this section, the virtual-power-based QC framework including the central summation rule of Beex et al. (2012a) is applied to two numerical examples to illustrate the computational gain and accuracy that can be achieved. The elastoplastic lattice model of section 2 is used in the examples which have a true multiscale character, since large-scale networks with local adaptations are considered. The examples are set up in a dimensionless form.

The main purpose of this section is to establish that the virtual-powerbased QC method is a viable approach for non-conservative (i.e. dissipa- 
tive) lattice models, the computational efficiency and and that it results in a considerable reduction in computing times at an acceptable loss of accuracy. For more detailed analysis of the approximations introduced in different QC strategies, in the conventional reversible, atomistic setting, we refer the reader to the studies of Miller and Tadmor (2009); Luskin and Ortner (2009); Dobson et al. (2010) and Yang et al. (2013). The central summation rule of Beex et al. (2012a) is used here is because of its fully nonlocal character and because it gives a significant better approximation of the governing equations than the other fully nonlocal summation rules such as the cluster summation rules (Knap and Ortiz, 2001; Eidel and Stukowski, 2009). However, the virtual-power-based QC approach may also be combined with alternative summation strategies and this may have an effect on accuracy and efficiency.

\subsection{Problem statement}

The lattice spacings in horizontal and vertical direction are set to 1 . The length of the diagonal trusses is thus $\sqrt{2}$. The lattice model contains $100 \times 100$ unit cells (20,402 DOFs). The parameters of the trusses' material behavior are presented in Table 1, corresponding to a purely elastic response up to a strain of $1 \%$. The use of these parameters results in a smooth increase of plastic deformation for strains larger than $1 \%$ (see ahead to the left image in Fig. 9 for the response of a unit cell).

Table 1: Dimensionless material parameters.

\begin{tabular}{|l|l|}
\hline$Y$ & 1 \\
\hline$A$ & 1 \\
\hline${ }^{o} \sigma$ & 0.01 \\
\hline$H$ & 10 \\
\hline$\alpha$ & 0.5 \\
\hline
\end{tabular}

In the numerical examples, a stiff region of $6 \times 6$ unit cells is introduced at the center of the lattice by modeling the trusses in this square substantially stiffer than the regular trusses. The Young's modulus of these trusses is therefore increased by a factor of 100 and a large initial yield stress is used so that no plastic deformation occurs in the stiff region. The local stiff 
region in the lattice model can be regarded as a hard particle or region inside a fibrous material. An example can for instance be the locally thermally bonded material investigated by Hou et al. (2009) or an electronic textile with a light-emitting-diode mounted on it (Beex et al., 2013).

As a result of the local stiff region, large displacement fluctuations are expected near this domain so that a fully resolved region is required in and around it. The displacement fluctuations in the remaining domain are expected to be significantly smaller so that coarse triangles can be used. This results in a true multiscale example that forms a representative test case for the virtual-power-based QC method.

The local influence of this stiff region on the lattice is investigated for uniform loading using periodic boundary conditions (Kouznetsova et al., 2001). It can thus be considered as a representative volume element in a periodic structure. To this end, the cross-sectional area of the trusses of which both lattice points are located on the model edges is only half of the area of the other trusses. Uniform deformation is applied in horizontal direction up to a strain of $10 \%$. The maximum strain is reached in 100 increments in the QC simulations as well as in the direct lattice computations. Note that in the direct lattice computation, used to evaluate the QC framework, the boundary conditions are directly applied to the lattice points (truss nodes), whereas they are applied to the reppoints only in the QC model.

\subsection{Computational efficiency}

Four different triangulations are used to investigate the smallest possible size of the fully resolved region that still gives sufficiently accurate results (see Fig. 4). The fully resolved region is centered around the central stiff region since this is the region of interest where significantly fluctuating displacements occur. The coarse domain is kept similar (i.e. a similar coarseness) in the four triangulations so that only the influence of the size of the fully resolved region is investigated. Another reason to keep the coarse domain as coarse as possible is that the refinement of the coarse domain leads to a relatively large extra number of reppoints and sampling points for only a limited increase of the accuracy (Beex et al., 2011).

The diagram in Fig. 4 shows that the number of reppoints and sampling points of the four triangulations is only $5-15 \%$ of the number of points used in direct lattice computations. The number of reppoints is a general, platform-independent measure for the computational effort to solve the governing equations in each iteration and the number of sampling points is a 

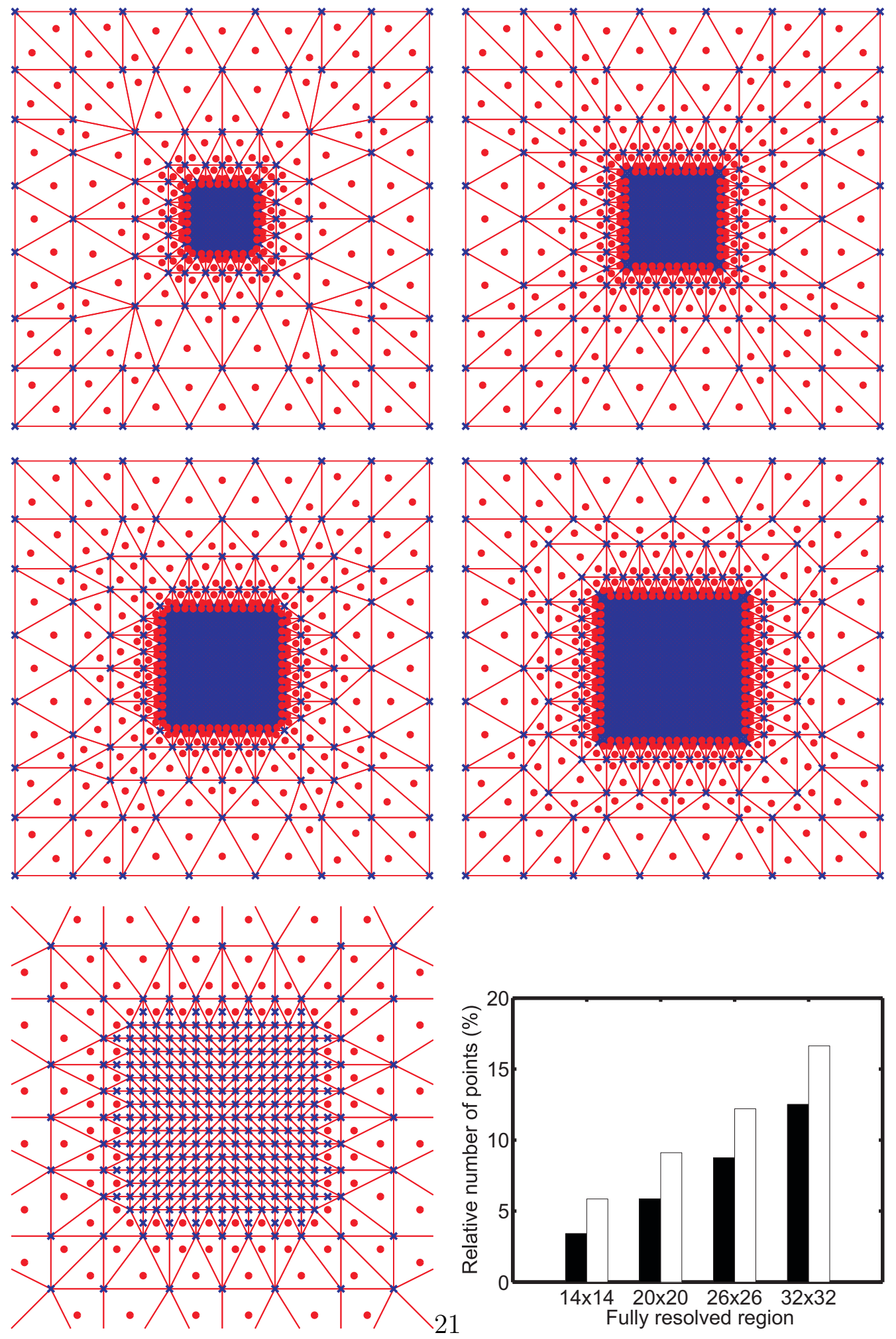

Figure 4: The four triangulations used to investigate the influence of a stiff region in the lattice. For clarity, trusses are not shown. Red circles represent internal sampling points $\left(w^{i} \geq 1\right)$ and blue crosses represent discrete sampling points $\left(w^{i}=1\right)$. The triangulations have fully resolved regions of $14 \times 14$ (top-left), $20 \times 20$ (top-right), $26 \times 26$ (middle-left) and $32 \times 32$ unit cells (middle-right). A zoom of the fully resolved region with $14 \times 14$ unit cells can be seen in the bottom-left image. The diagram on the bottom-right shows the number of reppoints (black bars) and sampling points (white bars) relative to the total number of points for the four triangulations. 
measure for the efforts to construct the governing equations in each iteration.

In Table 2 the computation times of a single increment for the different models are shown, which are average values based on the first ten increments. The values normalised by the increment time of the direct lattice computations correspond well to the relative number of reppoints and sampling points.

Table 2: CPU times to compute a single increment and the overhead times of the direct lattice computation (dlc) and the $\mathrm{QC}$ computations using triangulations with fully resolved regions of $14 \times 14$ lattice spacings (QC14), $20 \times 20$ lattice spacings $(\mathrm{QC} 20), 26 \times 26$ lattice spacings (QC26) and $32 \times 32$ lattice spacings (QC32).

\begin{tabular}{|l|l|l|l|l|l|}
\hline & dlc & QC14 & QC20 & QC26 & QC32 \\
\hline normalized increment $[-]$ & 1 & 0.03 & 0.06 & 0.12 & 0.16 \\
\hline increment $[s]$ & 192 & 5.8 & 10.9 & 22.4 & 31.2 \\
\hline Overhead $[s]$ & 0.4 & 3.2 & 3.7 & 4.2 & 5.0 \\
\hline
\end{tabular}

Hence, the relative number of reppoints and sampling points give a good indication of the achieved computational gain in computing times.

The overhead times are also presented in Table 2 (final line). For the direct lattice computation this includes the formulation of the lattice points, but for the QC implementations the interpolation functions need to be evaluated at the lattice points, sampling points have to selected from the lattice points, neighboring lattice points of the sampling points have to be selected and weight factors associated with the sampling points have to be computed. This overhead time has to be spent only once for each simulation and is generally smaller than the cost of a single increment.

The computational gain highly depends on the scale difference between the fully resolved domain and the model domain. In the reference case considered in this study, this scale difference is small, so that we are able to actually perform the direct lattice computation and compare its results to those of the QC computations. In practice, significantly larger scale differences are expected; consider e.g. the example of local failure in large-scale models, as in the study of Ha-Minh et al. (2011). In such cases, the relative number of reppoints and sampling points will be significantly smaller, which means that the computational gain will be significantly larger than that reported here. 
In Fig. 5 the relative number of reppoints and sampling points is presented for different model domains which all have a fully resolved region of $14 \times 14$ unit cells. The triangles in the coarse domain are of a similar size. It can be seen that the relative number of reppoints and sampling points reduces significantly for increasing model sizes and hence, a significant increase of the computational efficiency occurs for an increasing scale difference between the fully resolved domain and model domain. Note that the relative number of reppoints and sampling points approach their limit values which are the ratios that occur if no fully resolved region is present.
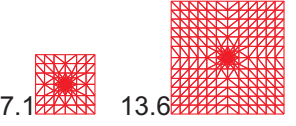

22.1
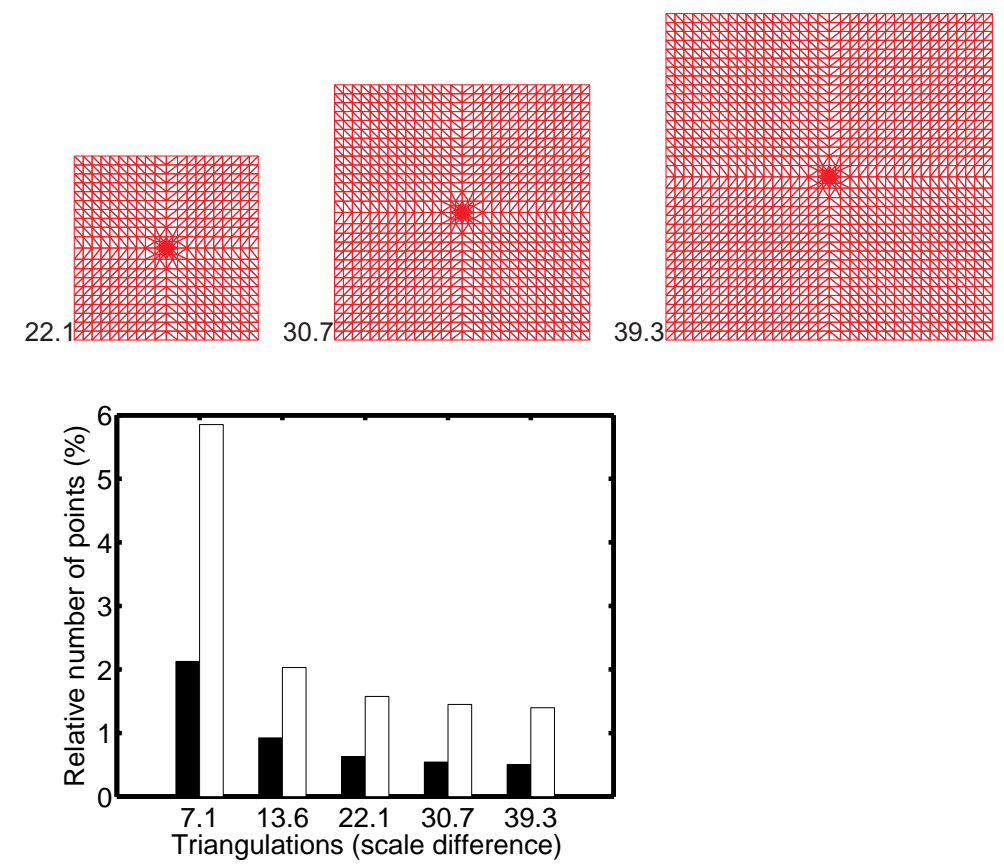

Figure 5: Top: five triangulations with a fully resolved region of $14 \times 14$ unit cells, but for different model domains. Sampling points are not shown. The numbers indicate the scale difference, which is defined as the ratio between the length of the entire model domain and the length of the fully resolved domain. Bottom: the relative number of reppoints (black) and sampling points (white) for the five triangulations.

\subsection{Accuracy}

Results of the direct lattice computation and QC simulation for the triangulation with a fully resolved region of $14 \times 14$ unit cells are presented in 
Fig. 6. The results of the trusses that are not shown in the top-right image of Fig. 6 belong to the summed lattice points. This means that they are represented by the internal sampling points.

Overall, the results predicted by the virtual-power-based QC method adequately correspond to those of the direct lattice computation for uniform deformation. The horizontal trusses on the left and right of the stiff region experience large plastic strains in tension, while the vertical trusses above and below the stiff region are plastically more compressed than the other vertical trusses. The trusses with the maximum plastic strains are located at the four corners of the stiff region.

To evaluate the accuracy of the $\mathrm{QC}$ results in a more quantitative manner, the plastic strains at maximum deformation of the horizontal trusses connected to the sampling points are regarded in more detail. The horizontal trusses are used for this because they are oriented in the loading direction. The error of the plastic strain in the truss $i, e^{i}$, is determined as follows:

$$
e^{i}=\left|\frac{{ }^{q c} z^{i}-{ }^{d l c} z^{i}}{d l c} z^{i}\right| \cdot 100 \%
$$

where $z^{i}$ is axial plastic strain in truss $i$. The superscript $q c$ refers to the solution of the virtual-power-based QC model for a particular triangulation and superscript $d l c$ refers to the solution of the direct lattice computation.

The errors of the plastic strains are presented in Fig. 7 for the four triangulations of Fig. 4. It is clearly visible that around the edges of the fully resolved regions rather large errors in the plastic strains are present. They correspond to errors observed for summation rules that lead to ghost-forces (Shenoy et al., 1999), but they are not true ghost-forces since the summation rule is fully nonlocal. Several global quantities (the average plastic strain, the total stored energy and the reaction forces on the right side of the entire model) were investigated to quantify the global error for the different triangulations, but they all correspond well with the global quantities computed with the full lattice model (less than $0.1 \%$ difference was observed). Hence, the QC computations with all four triangulations predict the global mechanical response well.

Locally however, the response is significantly different as can be seen in Fig. 7. Around the fully resolved regions the error is significant, but one is mainly interested in the fully resolved domain. Therefore, the error in 


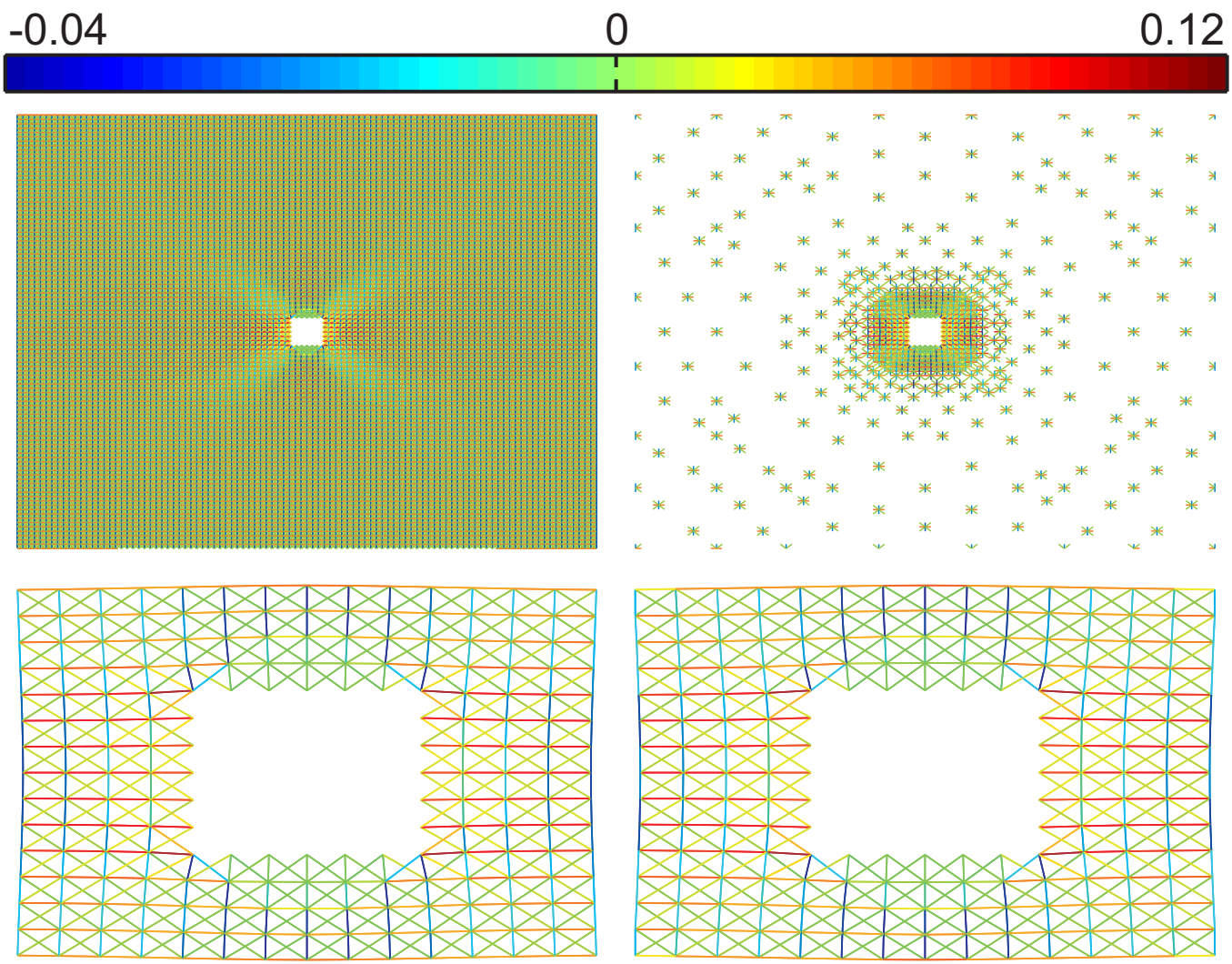

Figure 6: The plastic strains in the trusses at the maximum uniform deformation computed by the direct lattice computation (left) and the virtual-power-based QC computation (right) for the triangulation with a fully resolved region of $14 \times 14$ unit cells (see Fig. 4). The triangulation is not shown. The top images show the entire models and the bottom images show zooms of the fully resolved region around the stiff region (white).

the fully resolved domain is evaluated for the four triangulations based on the average error of the plastic strain in twelve lattice trusses. The twelve interactions of interest are shown on the left in Fig. 8 and again Eq. (32) is used for the error.

The local average relative errors of the plastic strains for the four triangulations shown in Fig. 4 are shown on the right in Fig. 8. The trend of the local average relative errors is decreasing for an increasing size of the fully resolved domain. A pronounced decrease is noticed between the error of the triangulation with a fully resolved domain of $14 \times 14$ unit cells and 


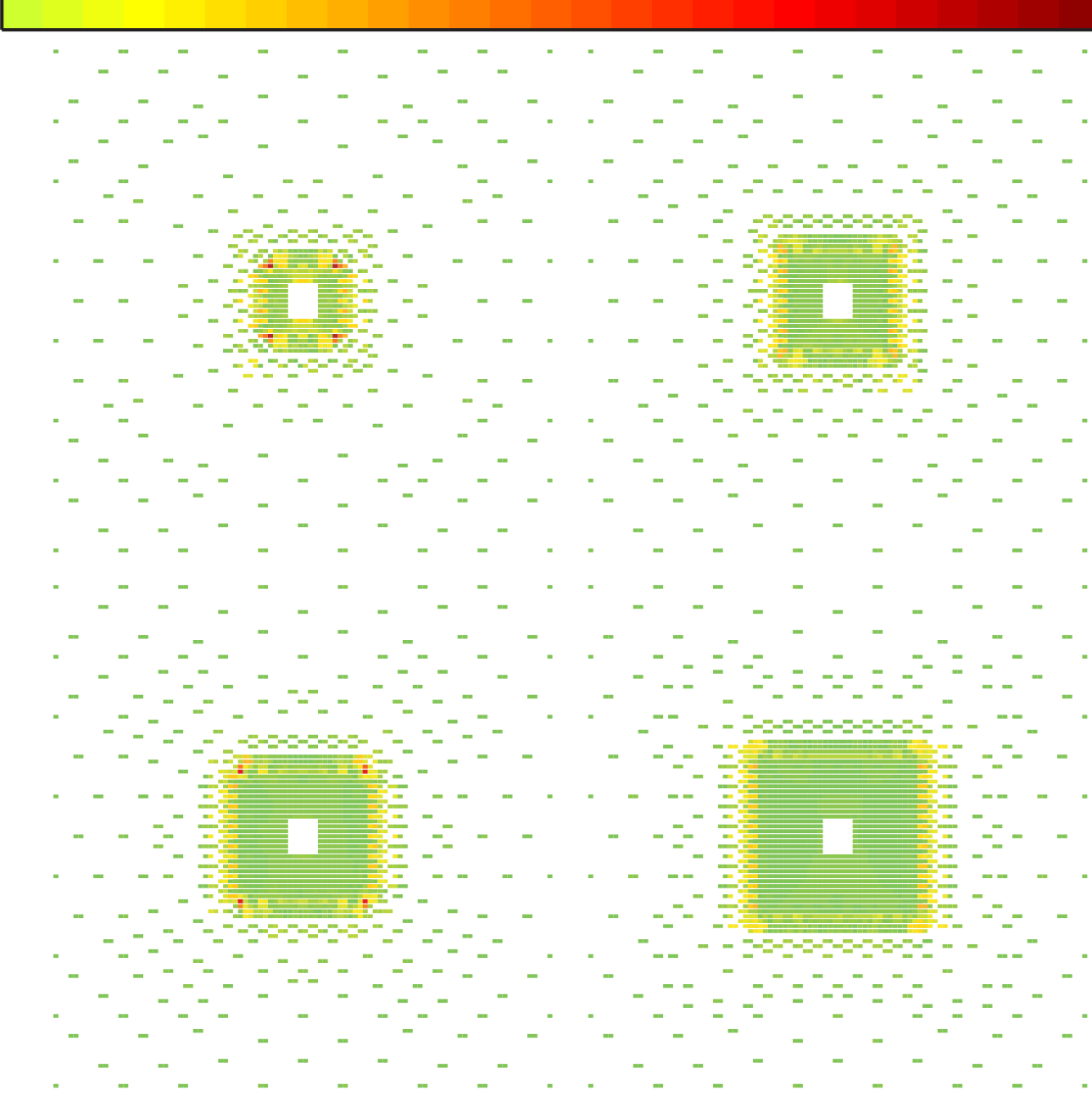

Figure 7: The errors of the plastic strains in the sampling points of the four triangulations of Fig. 4 plotted on the undeformed configurations.

the error of the triangulation with a fully resolved domain of $20 \times 20$ unit cells. For triangulations with fully resolved regions larger than $20 \times 20$ unit cells, a relatively small decrease can be observed. This is in agreement with the results of Beex et al. (2012a) in which the central summation rule is used for atomistic lattice models. In the results of Beex et al. (2012a), the error also decreases significantly if the size of the fully resolved domain is enlarged 
so that all local displacement fluctuations occur inside the fully resolved domain. For a further increase of the size of the fully resolved domain, only a minor decrease of the error was observed. In Beex et al. (2012a), it has been shown that if an error in the fluctuations field remains at the border of the fully resolved domain, the error remains non-zero. This is in agreement with the local average relative errors in Fig. 8.

The most important observation based on Fig. 8 is that for the triangulation with a fully resolved region of $20 \times 20$ unit cells, the local average relative error is below $5 \%$. This indicates that this triangulation gives results that may often be considered to be accurate enough, while the computational cost is low (see Fig. 4). A triangulation with a larger fully resolved region can be used if a larger accuracy is required, but this is at the expense of the computational efficiency.
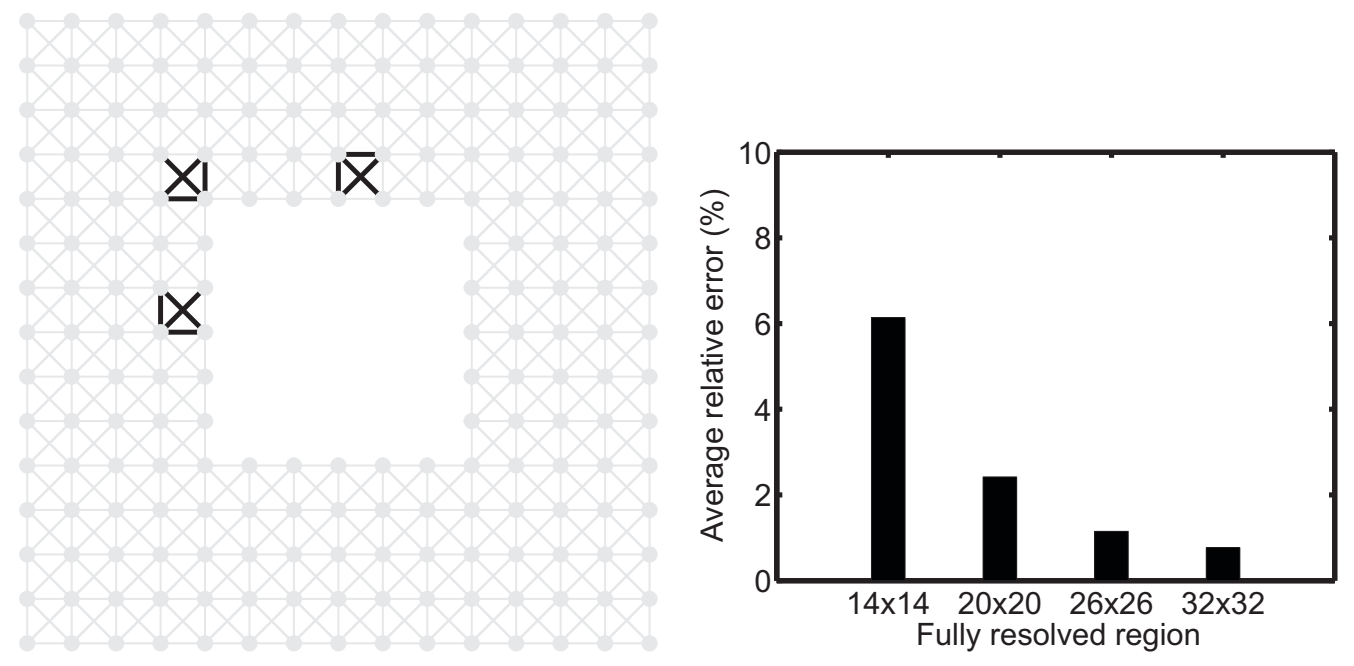

Figure 8: The twelve interactions selected around the stiff region (left) and (right) the local average relative errors of the plastic strains of the twelve interactions shown in the left image for the four triangulations shown in Fig. 4.

\subsection{Textile-like lattice model}

To illustrate that the virtual-power-based QC framework picks up trends if the lattice model is changed, the Young's modulus of the diagonal trusses is next decreased by a factor of 10. In this way the mechanical response of the lattice more or less corresponds to a general response of lattice models for 
woven fabrics (Sharma and Sutcliffe, 2004; Beex et al., 2013). In such lattice models, the relatively stiff horizontal and vertical interactions represent two families of yarns and the compliant diagonal trusses represent the rotational stiffness between the two families of yarns. Except for the stiffness of the diagonal trusses, all other model parameters and boundary conditions are the same as before. The stiff region in this case may represent a lightemitting-diode placed on a patch of electronic textile (Beex et al., 2013).

The homogenized stress-strain responses of a unit cell of the textile-like lattice model is compared to the response of the original lattice on the left in Fig. 9. The initial stiffness (during macroscopic uniform deformation) reduces to approximately $80 \%$ of the original stiffness. The initial stiffness during macroscopic shear deformation however decreases to approximately $10 \%$ of the original stiffness (not shown here). This is caused by the fact that during macroscopic shear deformation mainly the diagonal trusses are loaded, whereas during macroscopic uniform deformation the diagonal and vertical trusses are also loaded through the horizontal trusses.

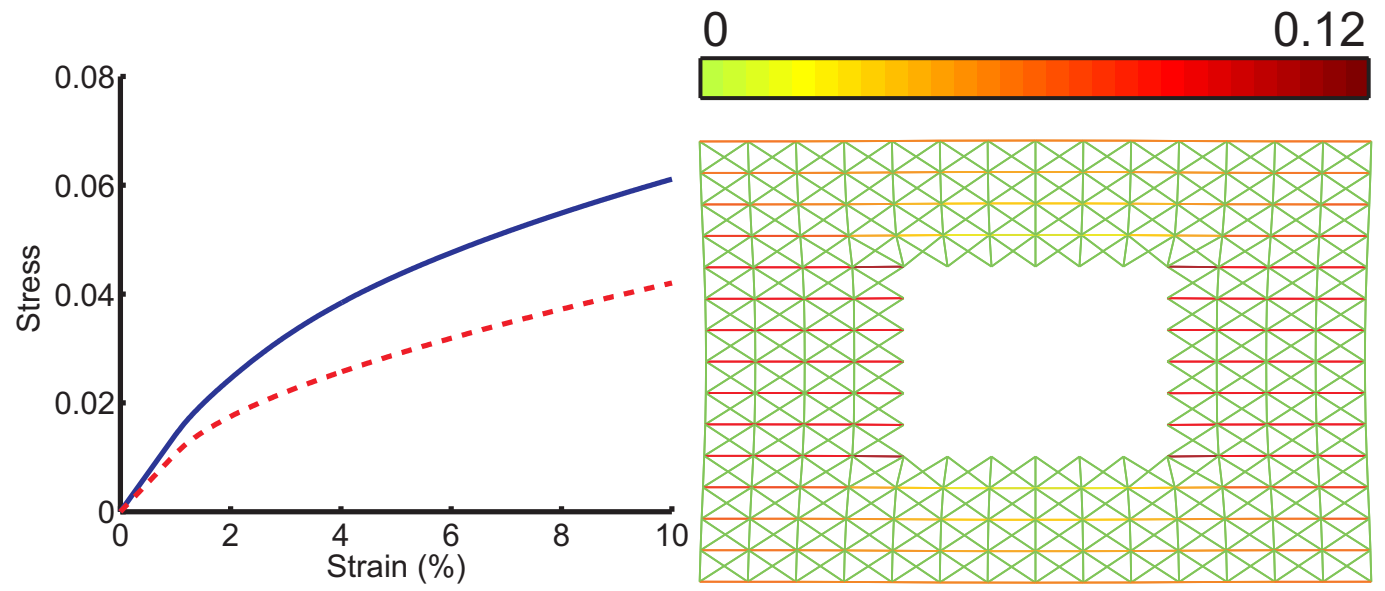

Figure 9: Left: the homogenized engineering stress-strain responses of an original unit cell (solid line) and of a unit cell with compliant diagonal interactions (dashed line) for uniform loading. Right: the plastic strains in the fully resolved region computed by the virtual-power-based QC model for the triangulation with a fully resolved region of $20 \times 20$ unit cells (at maximum applied uniform deformation).

The plastic strains predicted with the virtual-work-based QC framework for the lattice with compliant diagonal trusses are presented on the right in Fig. 9. They show a clear difference with the previous results. In this 
case only the horizontal interactions are plastically elongated. Because the diagonal interactions only yield at an axial strain of $10 \%$ (due to their small Young's modulus), no yield of the diagonal interactions can be observed at maximum uniform loading. Because the diagonal trusses only transfer small loads, the vertical trusses are also not plastically deformed. The virtualpower-based QC framework is thus well applicable a wide range of dissipative lattice models.

\section{Conclusion}

Lattice models and discrete networks are appropriate to describe the mechanical behavior of fibrous and heterogeneous materials, since they automatically incorporate mesoscale phenomena that dominate the material response of these materials. Large-scale computations of lattice models and discrete networks that are necessary for physically relevant simulations, involve substantial computational efforts since a large number of lattice points is required. The quasicontinuum (QC) method is a suitable multiscale approach to reduce the computational efforts of lattice models and discrete networks because it

- allows the incorporation of the mesoscopic lattice model in regions of interest,

- completely relies on the lattice model and not on accompanying continuum descriptions that can be complex to construct for fibrous materials and

- does not require a coupling or handshaking procedure (in the proposed framework).

Several QC methods presented so far, are based on energy-minimization and can thus only deal with conservative lattice models. Many lattice models for fibrous materials however, are non-conservative. In this paper, a virtualpower-based QC formulation is presented that can deal with non-conservative lattice models. Since it is based on the virtual-power statement, the construction of the governing equations is accurate in contrast to force-based QC methods which relate the internal forces incompletely to the virtual displacements of the lattice points. This is caused by the fact that in the virtual-power based QC formulation the virtual power is sampled (summed), 
instead of the forces. The dissipation involved in non-conservative lattice models is naturally incorporated by the constraints that follow from the virtual power statement. Another point of departure that is not used here could also be the so-called minimum principle (Ortiz and Stainier, 1999) in which the incremental work is minimized. Whether this approach would lead to a different framework remains to be seen.

In the numerical examples, two straightforward lattice models with elastoplastic trusses are used to show the possibilities of the method. The examples are truly multiscale in nature. Since no continuum descriptions are necessary and no internal interfaces occur, the QC counterparts of the direct lattice models are surprisingly easy to implement. The two elastoplastic lattice models show different results that are adequately captured the virtualpower-based QC framework. Comparisons with the results of direct lattice computations indicate that the local average relative error in the results of the virtual-power-based QC method are below $5 \%$ for a specific triangulation. Compared to the direct lattice simulations, the computational efficiency of the QC computation for this triangulation is $6 \%$ in terms of degrees of freedom and $9 \%$ in terms of effort to construct the governing equations (i.e. the number of sampling points). Since the considered problems are representative studies only, the computational gain is expected to be even larger for large-scale models in which a higher grade of scale separation is present. This makes the virtual-power based QC framework a powerful method to reduce the computational cost of dissipative lattice models while ensuring a high accuracy in regions of interest.

\section{Acknowledgements}

This research is supported by the Dutch Technology Foundation STW, which is the applied science division of NWO, and the Technology Programme of the Ministry of Economic Affairs under Project Nr. 10104.

\section{References}

Arnoux, P.J., Bonnoit, J., Chabrand, P., Jean, M., Pithioux, M., 2002, Numerical damage models using a structural approach: application in bones and ligaments, The European Physical Journal of Applied Physics, Vol. 17, 65-73. 
Beex, L.A.A., Peerlings, R.H.J., Geers, M.G.D., 2011, A quasicontinuuum methodology for multiscale analyses of discrete microstructural models, International Journal for Numerical Methods in Engineering, Vol. 86, 701718 .

Beex, L.A.A., Peerlings, R.H.J., Geers, M.G.D., 2012a, Central summation in the quasicontinuum method, Submitted.

Beex, L.A.A., Verberne, C.W., Peerlings, R.H.J., 2013, Experimental identification of a lattice model for woven fabrics: application to electronic textile, Composites: Part A, Vol. 48, 82-92.

Bronkhorst, C.A., 2003, Modelling paper as a two-dimensional elastic-plastic stochastic network, International Journal of Solids and Structures, Vol. 40, 5441-5454.

Chen, G., Baker, G., 2003, Influence of bond slip on crack spacing in numerical modeling of reinforced concrete, Journal of Structural Engineering, Vol. 129, 1514-1521.

Clyne, T.W., Markaki, A.E., Tan, J.C., 2005, Mechanical and magnetic properties of metal fibre networks, with and without a polymeric matrix, Composites Science and Technology, Vol. 65, 2492-2499.

Cusatis, G., Bažant, Z.P., Cedolin, L., 2003, Confinement-shear lattice model for concrete damages in tension and compression: 1. Theory, Journal of Structural Engineering, Vol. 129, 1439-1448.

Delincé, M., Delannay, F., 2004, Elastic anisotropy of a transversely isotropic random network of interconnected fibres: non-triangulated network model, Acta Materialia, Vol. 52, 1013-1022.

Desphande, V.S., Fleck, N.A., Ashby, M.F., 2001, Effective properties of the octet-truss lattice material, Journal of the Mechanics and Physics of Solids, Vol. 49, 1747-1769.

Dobson, M., Luskin, M., Ortner, C. 2010, Accuracy of quasicontinuum approximations near instabilities, Journal of the Mechanics and Physics of Solids, Vol. 58, 1741-1757. 
Doyoyo, M., Hu, J.W., 2006, Plastic failure analysis of an auxetic foam or inverted strut lattice under longitudinal and shear loads, Journal of the Mechanics and Physics of Solids, Vol. 54, 1479-1492.

Eidel, B., Stukowski, A., 2009, A variational form of the quasicontinuum method based on energy sampling in clusters, Journal of the Mechanics and Physics of Solids, Vol. 57, 87-108.

Fish, J., Nuggehally, M.A., Shephard, M.S., Picu, C.R., Badia, S., Parks, M.L., Gunzburger, M., 2007, Concurrent AtC coupling based on a blend of the continuum stress and the atomistic force, Computer Methods in Applied Mechanics and Engineering, Vol. 196, 4548-4560.

Germain, P., 1973, The method of virtual power in continuum mechanics Part 2: microstructure, SIAM Journal on Applied Mathematics, Vol. 25, $556-575$.

Gonella, S., Ruzzene, M., 2008, Homogenization of vibrating periodic lattice structures, Applied Mathematical Modelling, Vol. 32, 459-482.

Ha-Minh, C., Kanit, T., Boussu, F., Imad, A., 2011, Numerical multi-scale modeling for textile woven fabric against ballistic impact, Computational Materials Science, Vol. 50, 2172-2184.

Hatami-Marbini, H., Picu, R.C., 2009, An eigenstrain formulation for the prediction of elastic moduli of defective fiber networks, European Journal of Mechanics A/Solids, Vol. 38, 305-316.

Hou, X., Acar, M., Silberschmidt, V.V., 2009, 2D finite element analysis of thermally bonded non-woven materials: continuous and discontinuous models, Computational Materials Science, Vol. 46, 700-707.

Kato, S., Minami, H., Yoshino, T., Namita, T., 1997, Analysis of membrane structures based on fabric lattice model considering viscous characteristics, Proceedings of the IASS international symposium on shell spatial structures, Singapore.

Kim, H., Buttlar, W.G., 2009, Discrete fracture modeling of asphalt concrete, International Journal of Solids and Structures, Vol. 46, 2593-2604. 
Knap, J., Ortiz, M., 2001, An analysis of the quasicontinuum method, Journal of the Mechanics and Physics of Solids, Vol. 49, 1899-1923.

Kouznetsova, V., Brekelmans, W.A.M., Baayens, F.P.T., 2001, An approach to micro-macro modeling of heterogeneous materials, Computational Mechanics, Vol. 27, 37-48.

Kulkarni, Y., Knap, J., Ortiz, M., 2008, A variational approach to coarse graining of equilibrium and non-equilibrium atomistic description at finite temperature, Journal of the Mechanics and Physics of Solids, Vol. 56, 1417-1449.

Kwon, S., Lee, Y., Park, J.Y., Sohn, D., Lim, J.H., Im, S. 2009, An efficient three-dimensional adaptive quasicontinuum method using variable-node elements, Journal of Computational Physics, Vol. 228, 4789-4810.

Lilliu, G., Van Mier, J.G.M., 2003, 3D lattice type fracture model for concrete, Engineering Fracture Mechanics, Vol. 70, 927-941.

Luskin, M., Ortner, C., 2009, An analysis of node-based cluster summation rules in the quasicontinuum method, SIAM Journal on Numerical Analysis, Vol. 47, 3070-3086.

Miller, R., Ortiz, M., Phillips, R., Shenoy, V., Tadmor, E.B., 1998, Quasicontinuum models of fracture and plasticity, Engineering Fracture Mechanics, Vol. 61, 427-444.

Miller, R.E., Tadmor, E.B., 2002, The quasicontinuum method: overview, applications and current directions, Journal of Computer-Aided Materials Design, Vol. 9, 203-239.

Miller, R.E., Tadmor, E.B., 2009, A unified framework and performance benchmark of fourteen multiscale atomistic/continuum coupling methods, Modelling and Simulation in Materials Science and Engineering, Vol. 17, 053001 .

Mohr, D., 2005, Mechanism-based multi-surface plasticity model for ideal truss lattice materials, International Journal of Solids and Structures, Vol. 42, 3235-3260. 
Ortiz, M., Stainier, S., 1999, The varational formulation of viscoplastic constitutive updates, Computer Methods in Applied Mechanics and Engineering, Vol. 171, 419-444.

Ostoja-Starzewski, M., Wang, G., 2006, Particle modeling of random crack patterns in epoxy plates, Probabilistic Engineering Mechanics, Vol. 21, $267-275$.

Peng, X.Q., Cao, J., 2005, A continuum mechanics-based non-orthogonal constitutive model for woven composite fabrics, Composites: Part A, Vol. $36,859-874$.

Ridruejo, A., González, C., Llorca, J., 2010, Damage micromechanisms and notch sensitivity of glass-fiber non-woven felts: an experimental and numerical study, Journal of the Mechanics and Physics of Solids, Vol. 58, 1628-1645.

Rinaldi, A., Krajcinovic, D., Peralta, P., Lai, Y.C., 2008, Lattice models of polycrystalline microstructures: a quantitative approach, Mechanics of Materials, Vol. 40, 17-36.

Shenoy, V.B., Miller, R., Tadmor, E.B., Rodney, D., Phillips, R., Ortiz, M., 1999, An adaptive finite element approach to atomistic scale mechanics-the quasicontinuum method, Journal of the Mechanics and Physics of Solids, Vol. 47, 611-642.

Sharma, S.B., Sutcliffe, M.P.F., 2004, A simplified finite element model for draping of woven material, Composites: Part A, Vol. 35, 637-643.

Silberstein, M.N., Pai, C.L., Rutledge, G.C., Boyce, M.C., 2012, Elasticplastic behavior of non-woven fibrous mats, Journal of the Mechanics and Physics of Solids, Vol. 60, 295-318.

Simo, J.C., Taylor, R.L., 1986, A return mapping algorithm for plane stress elastoplasticity, International Journal for Numerical Methods in Engineering, Vol. 22, 649-670.

Stylianopoulos, T., Barocas, V.H., 2007, Volume-averaging theory for the study of the mechanics of collagen networks, Computer Methods in Applied Mechanics and Engineering, Vol. 196, 2981-2990. 
Tadmor, E.B., Ortiz, M., Philips, R., 1996a, Quasicontinuum analysis of defects in solids, Philosophical Magazine A, Vol. 73, 1529-1563.

Tadmor, E.B., Philips, R., Ortiz, M., 1996b, Mixed atomistic and continuum models of deformation in solids, Langmuir, Vol. 12, 4529-4534.

Ten Thije, R.H.W., Akkerman, R., Huétink, J., 2007, Large deformation simulation of anisotropic material using an updated Lagrangian finite element method, Computer Methods in Applied Mechanics and Engineering, Vol. 196, 3141-3150.

Wilbrink, D.V., Beex, L.A.A., Peerlings, R.H.J., 2013, A discrete network model for bond failure and frictional sliding in fibrous materials, International Journal of Solids and Structures, Vol. 50, 1354-1363.

Wilkins, M.L, 1963, Calculation of elastic-plastic flow, In: Balder, B. et al. (Eds.), Methods of Computational Physics 3, Academic Press, New York, 1964.

Xiao, B.K., Belytschko, T., 2004, A bridging domain method for coupling continua with molecular dynamics, Computer Methods in Applied Mechanics and Engineering, Vol. 193, 1654-1669.

Yang, Q., Biyikli, E., To, A., 2013, Multiresolution molecular mechanics: statics, Computer Methods in Applied Mechanics and Engineering, Vol. $258,26-38$.

Zeman, J., Peerlings, R.H.J., Geers, M.G.D., 2011, Non-local energetics of random heterogeneous lattices, Journal of the Mechanics and Physics of Solids, Vol. 59, 1214-1230.

Zhang, Y., Gunzburger, M., 2010, Quadrature-rule type approximations to the quasicontinuum method for long-range interatomic interactions, Computer Methods in Applied Mechanics and Engineering, Vol. 199, 648-659.

Zhao, X.H., 2012, A theory for large deformation and damage of interpenetrating polymer networks, Journal of the Mechanics and Physics of Solids, Vol. 60, 319-332.

Zohdi, T.I., Powell, D., 2006, Multiscale construction and large-scale simulation of structural fabric undergoing ballistic impact, Computer Methods in Applied Mechanics and Engineering, Vol. 195, 94-109. 